\title{
DEMANDA DE SECESIÓN EN CATALUÑA Y SISTEMA DEMOCRÁTICO. EL PROCÉS A LA LUZ DE LA EXPERIENCIA COMPARADA
}

\author{
ALBERTO LÓPEZ BASAGUREN \\ Catedrático de Derecho Constitucional \\ Universidad del País Vasco ${ }^{1}$
}

\section{SUMARIO}

I. El carácter ineludible de la comparación y sus límites. II. El «derecho a decidir» como enmascaramiento del derecho de autodeterminación. III. «Derecho a decidir» y referéndum sobre el estatus político. IV. Demanda secesionista, principio democrático y principio de legalidad: ¿tiene salida el laberinto?

\section{EL CARÁCTER INELUDIBLE DE LA COMPARACIÓN Y SUS LÍMITES}

El proceso político en torno a la demanda de secesión que se viene desarrollando en Cataluña desde hace aproximadamente un lustro, no es un proceso aislado; se inserta en un contexto en el que, en algunos países de nuestro entorno, en tiempos más o menos recientes, se han desarrollado procesos similares. En este sentido, el ejemplo tradicional ha sido el de la reclamación secesionista en Quebec, Canadá, al que se ha venido a sumar, muy recientemente, como es de sobra conocido, el caso de Escocia, en el Reino Unido. Desde hace mucho tiempo vengo sosteniendo que, guste o disguste, la reclamación secesionista en Cataluña estaba llamada a ser contrastada, de forma ineludible, con los procesos políticos seguidos en otros países democráticos; muy especialmente en un mundo tan inmediatamente interconectado como el de esta época.

He venido sosteniendo, en este sentido, que el contraste del proceso seguido en Cataluña con los seguidos en Quebec y en Escocia, principalmente, trasciende

1 Grupo de Investigación del Sistema Vasco IT743-13. Este trabajo ha sido realizado en el ámbito del Proyecto de Investigación DER 2013-47774-P, financiado por el Ministerio de Economía y Competitividad.

UNED. Teoría y Realidad Constitucional, núm. 37, 2016, pp. 163-185 
el ámbito en el que tradicionalmente se ha movido el Derecho comparado, para entrar en un terreno más exigente; el terreno de lo que el constitucionalista canadiense Sujit Choudhry ha denominado la migración de las ideas constitucionales, que, en su opinión, impone un método que ha denominado dialogical method, método dialogal o dialogístico ${ }^{2}$. Un método que obligaría a contrastar la forma de afrontar una cuestión o problema determinado en un sistema político democrático concreto con la forma de afrontar el mismo problema o cuestión por parte de los demás sistemas democráticos, a los efectos de contrastar la solidez de su respectiva fundamentación en los principios democráticos. En los últimos tiempos estamos asistiendo al desarrollo de un proceso de este tipo con la influencia que está teniendo la evolución del concepto de matrimonio en los sistemas democráticos, en relación con la aceptación del matrimonio entre personas del mismo sexo, lo que hasta hace muy pocos años era prácticamente inimaginable.

La necesidad de contraste de la propia experiencia con las experiencias que parecen haber desarrollado de forma más adecuada los principios que subyacen a los sistemas democráticos es ineludible, ulteriormente, por una razón práctica. Quienes impulsan los procesos secesionistas afirman que sus demandas se asientan, precisamente, sobre los mismos elementos que han sido desarrollados en aquellos sistemas considerados modélicos en la forma de afrontar democráticamente demandas de ese tipo. Cuando se contrasta lo que afirman con los presupuestos aceptados en aquellos sistemas políticos o en los estándares democráticos internacionales se descubre que lo que está en la base de esa justificación es una lectura parcial e interesada de aquellas experiencias; en muchos casos, ocultando elementos esenciales de aquellos estándares o, simplemente, manipulando lo que aquellas experiencias han establecido como elementos esenciales del proceso ${ }^{3}$.

En este sentido, el contraste entre la forma de afrontar las demandas secesionistas en España frente a la forma de afrontarlas en otros países puede poner en evidencia dos tipos diferentes de cuestiones. Obliga a diferenciar, poniendo en sus justos términos, los aspectos que responden a las singularidades constitucionales o de otro tipo de cada país de los que representan una aplicación especialmente consistente de los principios que subyacen a los sistemas democráticos.

Ciertamente, cada sistema político, cada país, tiene sus singularidades y responde a una tradición histórica y a un peculiar desarrollo de su sistema político. Pero es incuestionable que los sistemas democráticos se asientan sobre unos principios comunes que son los que permiten identificarlos como tales.

2 Cfr. S. Choudhry: «Migration as a new metaphor in comparative constitutional law», in S. Choudhry (ed.): The Migration of Constitutional Ideas, Cambridge, Cambridge UP, 2006.

3 Se trata de una actitud que ya puse de relieve hace años; vid. A. LóPEZ BASAGUREN: «Stéphane Dion y las verdades silenciadas de la experiencia canadiense», estudio introductorio a la edición española de $\mathrm{S}$. Dion: La política de la claridad. Discursos y escritos sobre la unidad canadiense, Alianza, Madrid, 2005, pp. 11-23. 
En relación con la cuestión que nos ocupa — las demandas de secesión de un territorio integrado en un Estado democrático- hay que tener en cuenta, por una parte, la tradición histórica de los sistemas democráticos y del Derecho internacional, que es incontestable en el rechazo de un derecho a la secesión, con la evolución que ponen de relieve — dentro de los límites que se precisaránexperiencias como las indicadas de Canadá y el Reino Unido. En definitiva, hay que analizar hasta qué punto los procesos seguidos en estos países son una excepción en el ámbito de los sistemas democráticos o, incluso, en su caso, siéndolo parcialmente, en qué sentido contienen elementos que responden de forma especialmente idónea a los principios sobre los que se asientan los sistemas democráticos.

En este sentido, como se tratará de argumentar en las páginas que siguen, la experiencia canadiense tiene una significación muy especial. Sin romper con la tradición de los sistemas democráticos, en el sentido de rechazar la existencia de un derecho la secesión - como manifestación, en el ámbito constitucional interno, de lo que en el ámbito del Derecho internacional se configura como derecho a la libre determinación-, establece, sin embargo, una obligación difícilmente objetable sobre la base de los principios que subyacen a un sistema democrático: la obligación de afrontar democráticamente las demandas de secesión. Una obligación que será exigible cuando la demanda de secesión cumpla unos requisitos democráticos que garanticen la concurrencia de un apoyo incontestablemente mayoritario a aquella por parte de la población del territorio.

Aquí reside, a mi juicio, el nuevo paradigma del tratamiento de las demandas de secesión en los sistemas democráticos que incorpora el proceso político canadiense. Un paradigma que transforma significativamente el modelo tradicional de los propios sistemas federales, del que la Guerra civil norteamericana es el referente histórico más claro, pero del que mantiene algunos elementos básicos trascendentales; principalmente, la inexistencia de un derecho a la secesión — de acuerdo al Derecho Constitucional—o a la autodeterminación — de acuerdo al Derecho internacional-.

No se trata, por tanto, como alguien pudiera pensar, de elevar a categoría incontestable, en cada uno de los elementos que lo integran, el proceso político seguido en Canadá o en el Reino Unido, que, para superar el test democrático, deberían reproducirse necesariamente en cualquier lugar y circunstancia, como si constituyera una especie de derecho natural en esta materia, que diferenciaría entre lo legítimo y lo ilegítimo democráticamente.

Es sintomático, en este sentido, la interpretación mecanicista que hacen algunos en España en relación, especialmente, con la conocida Ley de la Claridad, como si fuese el elemento indispensable que aporta la experiencia canadiense y constituyese el talismán indiscutible para afrontar democráticamente las demandas de secesión en cualquier sistema democrático. No comparto esa postura. Es probable que se esté confundiendo lo que podemos denominar teoría de la claridad, elaborada, en sus elementos básicos, por el Tribunal Supremo (TS) de Cana- 
dá en su Dictamen (Reference/Renvoi) sobre la secesión de Quebec $(1998)^{4}$, con Ley de la Claridad, creyendo que esta Ley recoge o condensa aquella teoría, con la que, por tanto, podría identificarse o a la que aquella podría reducirse. Es un error. La Ley de la Claridad es el resultado de la interpretación que el Parlamento federal canadiense — su Cámara baja — realiza del Dictamen del TS sobre la secesión de Quebec; una interpretación que es discutible y que, ciertamente, ha sido discutida - principalmente, aunque no únicamente, por la Assemblée nationale de Quebec- 5 , en la que, en mi opinión, no se condensan los elementos más importantes que Canadá ha aportado a la experiencia de los sistemas democráticos en relación con esta cuestión. Quienes defienden la importación directa de la Ley de la Claridad a España olvidan que aquella es el resultado de un proceso político concreto, en el que confluyen elementos que son consustanciales a los sistemas democráticos con otros que son particulares del sistema constitucional canadiense. Para tener posibilidades de éxito, España tiene que realizar su propio proceso político, del que deberá surgir la específica forma de afrontar democráticamente las demandas de secesión existentes. España debe encontrar su propia vía, coherente con los fundamentos de su propio sistema constitucional y de sus singularidades. Una vía que, sin duda, debe confrontarse, en esa vía de diálogo a la que se refería S. Choudhry, con las experiencias de otros sistemas democráticos que, desde el punto de vista de los principios sobre los que se edifican éstos, han sido capaces de afrontar muy satisfactoriamente retos similares.

En conclusión, la confrontación de las formas de afrontar un determinado reto en distintos sistemas en relación con la mayor o menor solidez de su enraizamiento en los principios que subyacen al sistema democrático es un momento ineludible, que debe ser, necesariamente, un test de idoneidad democrática. Pero esa confrontación debe hacerse discriminando entre los aspectos vinculados a las singularidades de cada sistema político y los que son acertada consecuencia de las exigencias de los principios democráticos.

\section{EL DERECHO A DECIDIR COMO ENMASCARAMIENTO DEL DERECHO DE AUTODETERMINACIÓN}

En el debate político que tiene lugar en España respecto a las demandas de secesión, los sectores políticos que las impulsan han acuñado un término, una idea, que ha sido un gran éxito de mercadotecnia política: el «derecho a decidir».

4 Cfr. Reference re Secession of Quebec [1998] 2 SCR 217, de 20 de agosto. Un análisis de la argumentación utilizada por el TS de Canadá en el referido Dictamen puede verse, entre otros, en A. López BASAGUREN: «Los referendos de secesión de Quebec y la doctrina del Tribunal Supremo de Canadá», en SÁEnz Royo, E. y Contreras Casado, M. (eds.): La participación política directa. Referéndum y consultas populares, Comuniter, Zaragoza, 2013, pp. 53-91.

5 Vid. A. López BASAguren: «Los referendos de secesión de Quebec...», cit. 
La puso en circulación, entre nosotros, el entonces lehendakari Juan J. Ibarretxe, como fundamento de su conocido plan, en la primera década del presente siglo, y ha sido usada como idea-fuerza o, aun más, como idea-talismán, por las fuerzas políticas que reclaman la independencia de Cataluña en esta segunda década.

El problema en torno a esta idea es que se usa con sentidos diferentes, por los mismos protagonistas, según los casos, las circunstancias o el momento. Por una parte, con carácter general, se usa como un sinónimo del derecho a la libre determinación. Tanto Ibarretxe como las fuerzas políticas catalanas sostienen que el derecho a decidir consiste en la capacidad de un determinado «pueblo» de determinar su estatus político en lo que se refiere a su mantenimiento como parte integrante del Estado del que forma parte y, en su caso, la configuración de su estatuto político en el seno de aquel. Una capacidad que incluye tanto la posibilidad de optar por la independencia como la de determinar el estatus político de esa comunidad dentro del Estado en el supuesto de que optase por mantenerse en su seno. El derecho a decidir así entendido tiene su fundamento en la condición del País Vasco o de Cataluña como «nación»; lo que, desde el punto de vista jurídico, se concretaría en el derecho a la libre determinación de los pueblos reconocido en los Pactos internacionales de Derechos (1966) ${ }^{6}$. Esta justificación se encuentra tanto en la fundamentación del plan Ibarretxe $e^{7}$ como en la justificación de la pretensión independentista del nacionalismo catalán ${ }^{8}$.

Ni Ibarretxe ni $C i U$ planteaban, inicialmente, que el ejercicio del «derecho a decidir» se concretase en una demanda de independencia, respectivamente, del País Vasco y de Cataluña. Mientras que Ibarretxe trataba de sacar adelante su proyecto de nuevo Estatuto Político de Euskadi ${ }^{9}, C i U$ planteaba iniciar el camino para la creación de «estructuras de Estado» ${ }^{10}$. Pero en el fondo de ambos planteamientos estaba la capacidad de sus respectivas comunidades para decidir su independencia de España ${ }^{11}$, especialmente en el caso de que su propuesta fuese recha-

6 El artículo 1(1) tanto del Pacto internacional de Derechos Civiles y Políticos como del de Derechos Económicos y Sociales, aprobados por la Asamblea General de la ONU, Resolución 2200 A (XXI), de 16 de diciembre de 1966, establecen: «Todos los pueblos tienen el derecho de libre determinación. En virtud de este derecho establecen libremente su condición política y proveen asimismo a su desarrollo económico, social y cultural».

7 Sobre la propuesta presentada por el lehendakari J. J. IBARRETXE en el Parlamento Vasco en septiembre de 2002, vid. A. López BASAguren: «Trasformazioni dello Stato autonomico e riforma dello Statuto del Paese Basco: tra rottura e "accomodamento a volontà"», en GAmBino, S. (ed.): Regionalismo e Statuti. Le riforme in Spagna e in Italia, Giuffrè, Milano, 2008, pp. 199 ss.

8 En el programa electoral con el que Convergència i Unió se presentó a las elecciones al Parlamento catalán en noviembre de 2012 figuraba expresamente recogida esta reconstrucción del «derecho a decidir»: vid. A. López BASAguren: «Scottish referendum and Catalan «consultation»: two experiences in the mirror», en S. Bory \& P. OTaOla (eds.): Autonomies et indépendences: le nationalisme au XXI' siècle, Éditions Connaissances et Savoirs, Paris (en prensa).

9 Sobre el Estatuto Político de Euskadi, vid. A. López BASAguren: «Trasformazioni...», cit., pp. 204 sigs.

10 Vid. A. López BASAguren: «Scottish referendum and Catalan “consultation”», cit.

11 Hay que tener en cuenta que la independencia no es la opción única a que, inevitablemente, aboca el derecho a la libre determinación de los pueblos de acuerdo con lo establecido en el apartado 2 de la Decla- 
zada. En el caso del plan Ibarretxe el desarrollo de la propuesta en esa dirección quedó frustrado, tras las elecciones al Parlamento Vasco en marzo de 2009, por la pérdida del Gobierno por parte del PNV y sus aliados en los Gobiernos tripartitos presididos por Ibarretxe. Pero esa ha sido la evolución del proceso político en Cataluña, que se ha concretado en una reclamación explícita de independencia, cuyos hitos son la «consulta» o "proceso participativo» realizado el 9 de noviembre (9-N) de 2014, las elecciones al Parlamento catalán, celebradas en septiembre de 2015, planteadas como elecciones «plebiscitarias» a favor de la independencia por parte de los partidos nacionalistas, y la Resolución del Parlamento de Cataluña de 9 de noviembre de 2015, comúnmente conocida como Resolución de desconexión con España ${ }^{12}$.

El nacionalismo, en consecuencia, es incapaz de superar la concepción de su sociedad de referencia como nación vinculada al ejercicio de la soberanía y a la titularidad del ejercicio del derecho a la libre determinación e incapaz de aceptar la pacífica consideración en el ámbito académico sobre la configuración del derecho a la libre determinación en el Derecho internacional y su inaplicabilidad a sociedades o comunidades como las que son objeto de referencia en estas líneas ${ }^{13}$. Una reconstrucción que sintetizó el TS de Canadá en el citado Dictamen sobre la secesión de Quebec ${ }^{14}$. Todo es inútil, llegándose a la paradoja de que se sostenga como fundamento de su reclamación del «derecho a decidir», simultáneamente, el derecho a la libre determinación, consecuencia de su condición de nación, y el Dictamen del TS de Canadá, en el que, sin embargo, explícitamente se niega la aplicabilidad de ese derecho a supuestos como los referidos ${ }^{15}$.

El nacionalismo puede, legítimamente, defender el objetivo político de lograr que se reconozca el «derecho a decidir» como derecho a la libre determinación, a pesar de que a esa pretensión pueda oponerse, en el mismo plano político,

ration on the Granting of Independence to Colonial Countries and Peoples, aprobada por la AG de NU, Resolution $1514(\mathrm{XV})$, de 14 de diciembre de 1960: «All peoples have the right to self-determination; by virtue of that right they freely determine their political status and freely pursue their economic, social and cultural development». Sobre esta cuestión, vid. A. Cassese (1995): Self-Determination of Peoples. A Legal Reappraisal, Cambridge: Cambridge UP.

12 Cfr. Resolució 1/XI del Parlament de Catalunya, sobre l'inici del procés polític a Catalunya com a conseqüència dels resultats electorals del 27 de setembre de 2015, en el Butlletí Oficial del Parlament de Catalunya, XI Legislatura, $\mathrm{n}^{\circ}$. 7, 9.11.2015. He analizado estas cuestiones en A. LópEZ BASAGUREN: «Regional Defiance and Enforcement of Federal Law in Spain. The claims for sovereignty in the Basque Country and Catalonia», en A. JAKab \& D. Kochenov (eds.): The Enforcement of EU Law against the Member States. Methods against Defiance, Oxford: Oxford University Press (en prensa).

13 Vid. A. López Basaguren: «Estado democrático y secesión de territorios. Un análisis comparado sobre el tratamiento democrático de las reclamaciones secesionistas», en Arregi Aranburu, J. (ed.): La secesión de España. Bases para un debate desde el País Vasco, Madrid: Tecnos, 2014, pp. 39-68.

14 El TS de Canadá concluye: «Quebec could not, despite a clear referendum result, purport to invoke a right of self-determination to dictate the terms of a proposed secession to the other parties to the federation»: Reference re Secession of Quebec, cit., par. 151.

15 Estos dos elementos incompatibles o contradictorios se recogen expresamente en la fundamentación del plan Ibarretxe: vid. A. López BASAGUREN: «Trasformazioni...», cit., pp. 202-203. 
su falta de encaje en la configuración del derecho a la libre determinación en el Derecho internacional y la falta de reconocimiento de un derecho a la secesión en los sistemas democráticos — salvo casos aislados y, podemos decir, marginales ${ }^{16}$ _. Planteado así, como pretensión política, el debate sobre el «derecho a decidir» se afrontaría en términos estrictos; es decir, como una reclamación cuya materialización quedaría sometida a los procedimientos legales establecidos para su incorporación al ordenamiento jurídico. Por el contrario, los defensores del «derecho a decidir» lo plantean como un derecho inherente, cuya existencia no puede ser puesta en entredicho, que está reconocido por el Derecho internacional y que, en cualquier caso, es una concreción indiscutible del principio democrático. Con ello tergiversan radicalmente la realidad y vician, desde su propio origen, el debate político que tendría que plantearse sobre la viabilidad, la conveniencia o, simplemente, el acuerdo o desacuerdo sobre su incorporación al sistema jurídico, en la medida en que el rechazo a su pretensión no es posible, salvo al precio de la descalificación. Poco importa que, como se ha dicho, no haya ningún sistema democrático en el mundo, mínimamente relevante, que acoja ese «derecho a decidir» en los términos planteados. Se impone como condición lo que debe constituir el objeto del debate y de la confrontación política, con lo que se hurta la posibilidad misma de uno y otra.

En los últimos tiempos se ha sostenido que se había producido una transformación sustancial de la configuración del derecho a la libre determinación en el Derecho internacional como consecuencia de la declaración unilateral de independencia (DUI) de Kosovo y el aval que la misma recibió en la Opinión consultiva emitida por el Tribunal Internacional de Justicia ${ }^{17}$. En el movimiento catalán por la independencia se sostiene que esa Opinión del TIJ avalaría su capacidad para realizar una DUI si España no aceptase la voluntad mayoritaria del electorado catalán a favor de la independencia.

Kosovo es un caso excepcional que encaja en la remedial secession contemplada en la Declaración sobre las Relaciones amistosas entre los Estados, de Naciones Unidas ${ }^{18}$, como excepción a la limitación de la aplicabilidad del derecho a la libre determinación en los pueblos sometidos a dominio colonial, establecida en la ya citada Resolution 1514 (XV). Excepción asentada sobre la vulneración repetida y

16 Los dos únicos casos a los que se suele hacer referencia son Etiopía y St. Kitts and Nevis, en las Indias Occidentales británicas. El artículo 39 de la Constitución de Etiopía, de 1994, establece en el párrafo 1 el «incondicional derecho a la auto-determinación» de cualquiera de las Naciones, Nacionalidades y Pueblos en Etiopía, «incluido el derecho a la secesión». Por su parte, la The Saint Christopher and Nevis Constitution Order 1983 establece en el art. 113, párrafo 1: «The Nevis Island Legislature may provide that the island of Nevis shall cease to be federated with the island of Saint Christopher and accordingly that this Constitution shall no longer have effect in the island of Nevis».

17 Cfr. International Court of Justice (ICJ): Advisory Opinion. Accordance with the International Law of the Unilateral Declaration of Independence of Kosovo, de 22.07.2010.

18 Cfr. Declaration on Principles of International Law concerning Friendly Relations and Co-operation among States in accordance with the Charter of the United Nations, UN GA, Resolution 2625 (XXV), de 24.10.1970. 
flagrante de los derechos humanos de los miembros de la comunidad albanesa, que constituía la casi totalidad de la población, por parte de las autoridades serbias. En esa línea, el acceso a la independencia de Kosovo ha tenido un cualificado respaldo de la sociedad internacional. La actitud de las autoridades serbias impidió cualquier solución razonable dentro de la salvaguarda del principio de integridad territorial del Estado (Serbia). La provincia fue puesta bajo administración civil y militar de $\mathrm{NU}^{19}$. El Secretario General de NU nombró un Representante Especial —el antiguo Presidente de Finlandia, Martti Ahtisaari-quien, inicialmente, presenta un plan de negociación a las autoridades serbias, que respetaba el principio de integridad territorial de los Estados, que Serbia rechazó. Lo que llevó al Representante Especial a proponer la independencia de Kosovo como única salida viable al conflicto. A pesar de ello, son conocidas las extraordinarias dificultades que está encontrando Kosovo para ser aceptado como un miembro de pleno derecho de la sociedad internacional, para el reconocimiento por otros Estados y para su incorporación a las organizaciones internacionales.

Es ese el contexto en el que el TIJ analiza la DUI de los representantes de la comunidad kosovar y su adecuación al Derecho internacional. A pesar de esos antecedentes, el TIJ en ningún momento afirma la legitimidad de la DUI por parte de los representantes kosovares. El Tribunal insiste, de forma reiterada, que no se le ha pedido que se pronuncie sobre la legitimidad de la DUI realizada por los representantes kosovares y, aún menos, sobre la legitimidad de realizar una acción similar por entidades pertenecientes a un Estado ${ }^{20}$; de donde deduce una conclusión que ha provocado muy duras críticas en el ámbito académico ${ }^{21}$ : a juicio del TIJ es plenamente posible que un acto determinado - como es el caso de una DUI- no viole el Derecho internacional sin que, necesariamente constituya el ejercicio de un derecho conferido por él ${ }^{22}$. Es decir, el limbo jurídico en el que un acto puede no ser ni ilegal ni legal. El Tribunal insiste en que ha sido consultado sobre la primera cuestión — si la UDI de Kosovo es contraria al Derecho internacional—, no sobre la segunda — si la misma es conforme al Derecho internacional— ${ }^{23}$.

19 Cfr. Resolución 1244 (1999), del Consejo de Seguridad de NU, de 10 de junio.

20 Cfr. ICJ: Advisory Opinion on Kosovo, cit., par. 56: «...the task which the Court is called upon to perform is to determine whether or not the declaration of independence was adopted in violation of international law (...) The Court is not required (...) to take a position on whether international law conferred a positive entitlement on Kosovo unilaterally to declare its independence or, a fortiori, on whether international law generally confers an entitlement on entities situated within a State unilaterally to break away from it».

21 Vid. las referencias bibliográficas críticas sobre la Opinión consultiva sobre Kosovo del TIJ en A. LópeZ BASAGUREN: «Estado democrático y secesión de territorios...», cit.

22 Cfr. ICJ: Advisory Opinion on Kosovo, cit., par. 56.

23 Íbidem. Una actitud que reiterará más adelante, insistiendo en que, aunque distintos participantes en el proceso han planteado directamente la cuestión del derecho de la población de Kosovo a crear un Estado independiente, por tratarse de un supuesto de remedial secession (par. 82), sin embargo, el TIJ sostiene que «it is not necessary to resolve these questions in the present case. The General Assembly has requested the Court's opinion only on whether or not the declaration of independence is in accordance with international law» (par. 83). 
Estas consideraciones ponen de relieve que la DUI de Kosovo no solo no es un antecedente que legitimaría una similar declaración por parte de los representantes del electorado de Cataluña. Lo que pone de relieve es que, incluso en una situación tan excepcional como la de Kosovo, en la que concurren las condiciones para que su caso sea considerado un supuesto de la remedial secession previsto en el Derecho internacional, las dificultades para reconocer el ejercicio del derecho a la libre determinación fuera de las situaciones de dominio colonial es extraordinariamente difícil y complejo, salvo que haya acuerdo entre las partes.

\section{III. «DERECHO A DECIDIR»Y REFERÉNDUM SOBRE EL ESTATUS POLÍTICO}

En ocasiones, «derecho a decidir» se utiliza con otro significado más limitado; el de la capacidad de convocar un referéndum en el que los ciudadanos puedan manifestar su voluntad en relación con el «estatuto» político de esa comunidad. Tanto en el plan Ibarretxe como en el proceso de reclamación de la independencia en Cataluña la reclamación de convocatoria de un referéndum se configura como el instrumento capital del «derecho a decidir». Un referéndum que no necesariamente tiene que plantear la opción de la independencia — como no lo hacía la «consulta» que pretendía el Gobierno de Ibarretxe-, pero que, en cualquier caso, implica la aceptación de la capacidad de la comunidad política territorial para decidir sobre su futuro político, sin cortapisas, incluida la independencia, sea cual sea la opción que sus instituciones consideren conveniente plantear en cada momento. Es decir, aunque en ocasiones «derecho a decidir» se utiliza en el sentido de derecho a convocar un referéndum sobre el estatus político de una determinada comunidad, esa comprensión no queda limitada a la posibilidad de realizar el referéndum, sino que, de forma implícita, en la misma se contiene la idea de «derecho a decidir» como derecho a la libre determinación del estatus político, en la medida en que a la capacidad de realizar el referéndum se vincula la obligatoriedad del Estado de dar plena efectividad a la voluntad expresada por el electorado en el mismo.

\section{A) Referéndum, principio democrático y exigencia de previsión legal}

Planteado el «derecho a decidir» como capacidad de convocar un referéndum sobre el estatus político de la comunidad, sus defensores no se plantean siquiera el problema de si esa capacidad puede estar legítimamente limitada legalmente. Ciertamente, sus promotores consideran que es una consecuencia ineludible del principio democrático, entendido como un «valor absoluto», que prevalece sobre todos los demás principios constitucionales, incluido el principio de legalidad, como sostuvo $C i U$ en el programa con el que se presentó a las elecciones al Parlamento cata- 
lán en $2012^{24}$. Una convicción de legitimidad que llevó a tratar de sortear la falta de previsión legal de un referéndum similar a través de la pretensión de transformarlo en «consulta», manteniendo las características sustanciales del referéndum ${ }^{25}$.

El recurso al referéndum se ha ido extendiendo en los últimos tiempos, especialmente en aquellos sistemas, como los de origen británico, en los que era una figura realmente extraña, por el apego al principio tradicional de la soberanía parlamentaria, sobre una base indudable de ecos de democracia directa, que encuentran, en principio, y de forma generalizada, una favorable acogida ${ }^{26}$. Pero eso no puede llevar a una visión idealizada del referéndum, como instrumento insuperable de expresión democrática, eludiendo los peligros para el sistema democrático que pueden agazaparse tras la institución del referéndum, como pone en evidencia la historia de los sistemas políticos. Esto es lo que atribuye tanta importancia a la regulación legal del referéndum y a las limitaciones con que muchos sistemas jurídicos lo regulan, condicionados por su propia experiencia histórica. En este sentido, España es un país que ha tenido una mala experiencia que llevó al constituyente a regularlo de forma muy restringida. Una regulación legal que no es posible eludir, simplemente, apelando al principio democrático, cual bonne à tout faire, que permitiría, a conveniencia, determinar las reglas aplicables en cada situación, al margen de la interpretación de ese principio realizada en sede constituyente y legislativa.

La limitación de la utilización de referéndum dentro de la legalidad, como condición de garantía democrática es lo que, precisamente, respalda la Comisión de Venecia, del Consejo de Europa (CoE), en su Informe sobre el código de buenas prácticas en materia de referéndum ${ }^{27}$, en el que hace expresa referencia a la exigencia de que el uso del referéndum se haga de acuerdo con la legalidad, quedando excluidos cuando la Constitución o la Ley, de acuerdo con aquella, no lo prevean $^{28}$. Es decir, la Comisión de Venecia acepta como plenamente legítimas las

24 Convergència i Unió se presentó a las elecciones al Parlamento de Cataluña en 2012 con un programa en el que afirmaba que apostaban por el «derecho a decidir» para lograr el autogobierno que el pueblo de Cataluña reclama y necesita para vivir mejor «sin otra herramienta que la democracia», entendida como «un valor absoluto»: vid. Convergència i Unió: Programa electoral 2012. Catalunya 2020.

25 Eso es lo que planteó el lehendakari Ibarretxe con la Ley de la consulta, aprobada por el Parlamento Vasco y ese es el recorrido realizado en el proceso catalán por la independencia, que llegó, finalmente, a transformar la «consulta» en "proceso participativo» cuando la primera quedó suspendida al ser impugnada ante el TC. Pero se trata de una cuestión que ha resuelto ya reiteradamente el TC en una dirección que, en sus líneas básicas, considero correcta: vid. A. López Basaguren (2009): «Sobre referéndum y Comunidades Autónomas. La ley vasca de la "consulta" ante el Tribunal Constitucional (Consideraciones con motivo de la STC 103/2008)», en Revista d'Estudis Autonòmics i Federals, n. ${ }^{\circ}$ 9, 2009, pp. 202-240, así como A. López Basaguren: «Regional Defiance...», cit.

26 Sobre este proceso y sus fundamentos, vid. S. TIERney (2012): Constitucional Referendums. The Theory and Practice of Republican Deliberation, Oxford: Oxford UP.

27 Cfr. European Commission for Democracy Through the Law (Venice Commission): Code of Good Practice on Referendums —CDL-AD(2007)008_, Strasbourg, 119.03.2007.

28 En efecto, el Informe de la Comisión de Venecia afirma, de forma reiterada, el requisito de la legalidad. Así, afirma que «(t)he use of referendums must comply with the legal system as a whole, and especially 
restricciones a la convocatoria de referéndums, sin considerar que, con ello, se vulnere el principio democrático. Lo ha afirmado expresamente su Opinión sobre el referéndum en Crimea $^{29}$, en la que expresamente afirma que si la Constitución de Ucrania no permite referéndums sobre la secesión, ello no contradice de ninguna forma los estándares constitucionales europeos, pues, por el contrario, es típico de las Constituciones de los Estados miembros del CoE no permitir la secesión.

Sorprende que estas consideraciones de la Comisión de Venecia no hayan merecido ninguna reflexión por parte del Consell, sobre todo, cuando se trata de un Código que el organismo catalán utiliza en su primer Informe, sobre la consulta ${ }^{30}$, al que se hará reiterada referencia en las líneas que siguen. El subterfugio de cambiar la denominación de referéndum por «consulta» o por «proceso participativo» no altera el carácter sustancial del límite de la exigencia de previsión legal.

Es evidente que, en relación con esta cuestión, hay diferentes opciones constitucionales o legales. Hay países en los que la Constitución y la Ley guardan silencio respecto al referéndum, como ocurre, en general, en los sistemas de origen británico, por su propia tradición constitucional. En ellos es más fácil impulsar la realización de un referéndum, tanto a nivel nacional como regional, porque la ausencia de regulación constitucional supone ausencia de límites a ese nivel, permitiendo al legislador, estatal o regional, adoptar las medidas que permitan su convocatoria $^{31}$. Esto es, precisamente, lo que ocurrió en Canadá en relación con la convocatoria por parte de la Assemblée nationale de Quebec de los referéndums de 1980 y de 1995: el Parlamento provincial pudo hacerlo sin objeción constitucional por el silencio de la Constitución canadiense sobre la cuestión del referéndum. Pero, por el contrario, en el Reino Unido, a pesar de su singularidad cons-

the procedural rules. In particular, referendums cannot be held if the Constitution or a statute in conformity with the Constitution does not provide for them, for example where the text submitted to a referendum is a matter for Parliament's exclusive jurisdiction»: cfr. European Commission for Democracy Through the Law (Venice Commission), cit., apartado III.1:. The principle of the sovereignty of the people allows the latter to take decisions only in accordance with the law». En el mismo sentido, en el Explanatory Memorandum que acompaña al Informe, se reitera que «(t)he use of referendums must be permitted only where it is provided for by the Constitution or a statute in conformity with the latter, and the procedural rules applicable to referendums must be followed. On the other hand, referendums must be organised where the legal system provides for them (par. 26). Y, más adelante, precisa que «(u)nder the principle of the rule of law, the people are not exempt from compliance with the law. This applies to both procedural aspects and the substance of texts put to the vote, which must comply with all superior law. Legislative referendums must therefore comply with the Constitution; referendums within federated or regional entities must comply with the law of the central State» (par. 32). Para concluir afirmando que «(i)n order to prevent unlawful referendums, texts that are procedurally or substantively invalid must not be put to a referendum» (par. 34).

29 Cfr Cfr. European Commission for Democracy Through the Law (Venice Commission): Opinion on "Whether the decision taken by the Supreme Council of the Autonomous Republic of Crimea in Ukraine to organise a Referendum on becoming a Constituent Territory of the Russian Federation or restoring Crimea's 1992 Constitution is compatible with Constitutional Principles», Opinion no. 762 / 2014, de 21.03.2014 [CDL-AD(2014)002], par. 17.

30 Cfr. Consell Assessor per a la Transició Nacional (2013): La consulta sobre el futur polític de Catalunya, de 25 de julio.

31 Vid., sobre esta mayor facilidad de abrir la vía a la realización del referéndum en los sistemas en los que no hay regulación constitucional, en S. Tierney: Constitucional Referendums..., cit., pp. 137 sigs. 
titucional, en la que rige — a pesar de su evidente deterioro práctico - el principio de soberanía parlamentaria, en un sistema que carece de Constitución codificada y en el que ésta carece de supremacía sobre la legislación ordinaria, se entendió que el Parlamento de Escocia carecía de capacidad para convocar un referéndum en un tema - la Unión de los Reinos de Inglaterra y Escocia- que, como reserved matter, estaba vedada a su competencia por la Scotland Act ${ }^{32}$. Y solo fue posible convocar el referéndum una vez que, con la autorización del Parlamento británico, el Gobierno aprobó la Order in Council que reformaba la Scotland Act para atribuir expresamente esa competencia al Parlamento escocés ${ }^{33}$. Es decir, respeto del principio de legalidad, tanto en Quebec como en Escocia, aunque en Canadá no plantease un problema de legalidad y en el Reino Unido la reforma de esta fuese relativamente sencilla en el plano formal, una vez que concurría la decisión política de llevarla a cabo ${ }^{34}$.

Por el contrario, los límites de la legalidad para el Consell Assessor y para los impulsores de la secesión de Cataluña parece ser constituir un obstáculo que, por su carácter contrario al principio democrático, es fácilmente eludible con la simple apelación de aquel principio.

Los impulsores del referéndum sobre la secesión lo consideran el instrumento de expresión democrática supremo e incuestionable. Olvidan, sin embargo, que en el sistema político hay otras formas de expresar el respaldo social a la independencia. Como señala el TS de Canadá, ciertamente, el referéndum puede ser un instrumento de constatación de esa voluntad. Pero no necesariamente el único. Esa manifestación de voluntad no se impide aunque el referéndum no esté previsto en un determinado sistema. Cuestión distinta es la idoneidad del referéndum para la ratificación de la solución que finalmente se adopte, sea la secesión o, en su caso, cualquier otra alternativa.

\section{B) Referéndum y exigencia de requisitos cualificados}

En relación con el referéndum hay otro tema, de considerable importancia en el proceso político de reclamación de la independencia de Cataluña, que debe ser objeto de contraste con la experiencia de procesos similares seguidos en otros países. El Consell Assessor per a la Transició Nacional, en el primer Informe al que

32 Sobre el significado de las reserved matters en la Scotland Act, vid. A. PAGE: Constitutional Law of Scotland, Edinburgh: W. Green-Thomson Reuters, 2015, pp. 114 y sigs.

33 Sobre esta cuestión, puede verse A. López BASAGUREN: «Escocia: referéndum sobre la independencia y propuesta de reforma de la Devolution», en Revista Española de Derecho Constitucional, 2015, n . 104, pp. 135-167; J. Tornos Mas: De Escocia a Cataluña. Referéndum y reforma constitucional, Madrid: Iustel, 2015, pp. 53 y sigs.

34 Sigue teniendo pleno el análisis precoz de J. E. Murkens: Scottish Independence. A Practical Guide, Edinburgh: Edinburgh UP, 2007, pp. 50 y sigs. 
se viene haciendo referencia en estas líneas aborda la cuestión de los requisitos de mayoría y, en su caso, de participación, que serían exigibles en un referéndum de estas características. En muy pocas líneas el Consell resuelve fácilmente esta cuestión: «l'estudi comparat demostra que la regla absolutament general i acceptada como bona practica per il Consell d'Europa és la de la no exigència de quòrum de participació i la majoria simple dels vots emessos. Aquestes regles es corresponen amb les exigències del principi democràtic $i$ asseguren claredat en l'interpretació e implementació dels resultats. Exigir, en canvi, majories especials o reforçades soposa una excepció notable del principi democràtic i, a més, pot derivar en situacions complexes i problemàtiques» ${ }^{35}$.

Sorprende, de nuevo, la simplista argumentación del Consell, que elude lo sustancial del problema planteado, refugiándose, aparentemente, tras el Informe de la Comisión de Venecia. Los referéndums que son objeto de tratamiento en el Código de buenas conductas parecen, a todas luces, lo que podríamos denominar referéndums de legalidad; es decir, en relación con la adopción de decisiones políticas ordinarias y no decisiones «constituyentes»; y, mucho menos, decisiones que ponen en cuestión principios fuertemente asentados en el orden internacional. Esto es lo que pone de relieve el posterior informe de la misma Comisión de Venecia sobre el referéndum de secesión de Montenegro. En este Informe, la Comisión subraya, precisamente, la existencia en estas cuestiones de un hecho trascendental que debe ser tenido en cuenta: que el referéndum está relacionado con la «crucial cuestión» de la independencia del país y no cualquier decisión política ordinaria, por muy importante que pueda ser políticamente ${ }^{36}$. La consecuencia del carácter trascendental de la cuestión a decidir — la independencia del país — supone a juicio de la Comisión de Venecia, que, en primer lugar, sea legítimo exigir un mínimo de participación de electorado para dar validez a los resultados del referén$\mathrm{dum}^{37} ; \mathrm{y}$, en segundo lugar, que sea legítimo exigir un nivel mínimo de apoyo a

35 Cfr. Consell Assessor per a la Transició Nacional, cit., p. 184; aunque, finalmente, acepta que «per el cas que es vulgui assegurar especialment la legitimitat política del resultat» se podría aceptar un «quòrum de participació»: íbid., p. 185.

36 cfr. European Commission for Democracy Through the Law (Venice Commission): Opinion on the compatibility of the existing legislation in Montenegro concerning the organisation of Referendums with applicable International Standards, Opinion no. 343 / 2005 -CDL-AD(2005)041, Strasbourg, 19.12.2005. Ciertamente, "the issue at stake is possibly the most important decision that a political community may take by democratic means: its independence» (par. 24); lo que significa que «it has to be taken into account that the proposed referendum is one dealing with the crucial issue of the independence of the country» (par. 26). En la Opinión sobre el referéndum de Crimea, cit., par. 25, señala que la cuestión «concerns an issue of outstanding importance».

37 cfr. European Commission for Democracy Through the Law (Venice Commission): Opinion on Montenegro, cit. par. 22, afirma que «taking into account both comparative constitutional material and requirement of legitimacy in the light of the concrete circumstances in Serbia and Montenegro, the Commission concludes that the requirement that the majority of the electorate voted in the referendum for it to be valid is consistent with international standards» —aunque reconoce que hay países en los que, incluso, se exige un nivel de participación más elevado (par. 26) _. Ello es así, porque «the matter requires the broadest possible commitment of the citizens to the resolution of the issue» (par. 24). Tienen mucho interés, en relación con la legitimidad de exigir un quórum mínimo de participación, las observaciones que el Informe hace en el párra- 
la opción favorable a la independencia, superior a la mayoría simple de los votos emitidos, para darle validez, porque, en esa cuestión, precisamente, es necesario otorgar al resultado del referéndum una legitimidad incuestionable ${ }^{38}$.

Sorprende, realmente, que el Consell no tenga en cuenta el Informe de la $C_{0}$ misión de Venecia sobre Montenegro y se limite a utilizar, únicamente, el Código de buenas conductas, cuando el criterio de la especialidad hace que aquel sea el singularmente relevante en el tema que ocupaba su Informe. Y sorprende que, habiendo utilizado profusamente el Dictamen del TS de Canadá sobre la secesión de Quebec para legitimar el «derecho a decidir», eluda confrontarse con su construcción de la teoría de la claridad, siendo, como es, una de las aportaciones más importantes de su construcción doctrinal, igualmente utilizado por la Comisión de Venecia como una de sus referencias en el Informe sobre Montenegro ${ }^{39}$. Como es sabido, uno de los elementos cruciales de la doctrina del TS canadiense sobre el tratamiento democrático de las demandas de secesión consiste, precisamente, en el hecho de que, para que las instituciones de la Federación canadiense tengan la obligación de no permanecer indiferentes ante la demanda de secesión de la población de un determinado territorio debe concurrir una condición: que la demanda de secesión sea respaldada por una «clara mayoría» en sentido cualitativo, en respuesta a una pregunta clara, carente de ambigüedades, sobre ella ${ }^{40}$. Es esta exigencia de una clara mayoría desde el punto de vista cualitativo la que el Consell pretende hacer creer que no está ampliamente aceptada en el mundo democrático.

Los dos únicos casos que se suelen citar de Constituciones democráticas en las que se reconoce el derecho a la secesión de territorios internos, abundan en la misma exigencia de requisitos cualificados en el apoyo a la misma. La Constitu-

fo 27: «In short, the present requirement in the Law on Referendums is in conformity with international standards. However, the Commission makes the following observations on this matter: (a) the higher the level of participation, the more political authority will be attached to the result of the referendum, both inside and outside Montenegro; (b) in present circumstances any departure from the present requirement that a majority of the electorate should have voted should be made only with the agreement of the main political forces in Montenegro; and (c) to abandon or to reduce the present requirement, even with the agreement of the main political forces, would be likely to weaken the authority of the result of the referendum.»

38 En efecto, la Comisión de Venecia —European Commission for Democracy Through the Law (Venice Commission): Opinion on Montenegro, cit. par. 33- constata que esa exigencia de un apoyo superior a la mayoría simple de los votos es común en el ámbito internacional, precisamente, en el ámbito de los referéndums de auto-determinación, ámbito en el que tienen vigencia las «normas más exigentes». Y concluye: «While therefore the absence of any requirement of a specific level of support for a referendum on independence is not inconsistent with internationally recognised standards, the Commission emphasises that there are reasons for requiring a level higher than a simple majority of those voting, since this may be necessary to provide legitimacy for the outcome of a referendum» (par. 36). La Comisión elude pronunciarse por cuál de las opciones posibles para exigir una apoyo cualitativamente mayoritario es más idónea (par. 37). La posición mantenida en el Informe sobre Montenegro es reiterada por la Comisión de Venecia en el Informe sobre el referéndum de secesión de Crimea: Opinion on Crimea, cit.

39 Cfr. European Commission for Democracy Through the Law (Venice Commission): Opinion on Montenegro, cit.

40 Cfr. Reference re Secession of Quebec, cit., par. 87; vid., sobre esta cuestión, A. López BASAGuren: «Los referendos de secesión de Quebec...», cit. 
ción de Etiopía establece un procedimiento complejo, en el que es necesaria la aprobación de la iniciativa por una mayoría de dos tercios de los miembros de la Asamblea Legislativa de la «Nación, Nacionalidad o Pueblo», la posterior aprobación por un «voto mayoritario» en un referéndum convocado al efecto por el Gobierno Federal, la transferencia de los poderes por parte de éste y que se haya procedido a la división de los bienes de acuerdo a lo previsto en la Ley (art. 39.4). Por su parte, la Constitución de St. Kitts and Nevis establece que la isla de Nevis podrá dejar de estar federada con St. Christopher para lo que se exige el respaldo de los dos tercios de los miembros de su Asamblea y la aprobación en referéndum por al menos los dos tercios de los votos válidamente emitidos, previo depósito durante al menos seis meses en la referida Asamblea del proyecto de futura Constitución de la Isla de Nevis.

Es realmente sorprendente que el Consell pretenda ignorar todo ello, tratando de hacer creer que su postura encuentra respaldo indiscutible en el ámbito democrático internacional, utilizando pronunciamientos importantes de altos tribunales a conveniencia, ignorándolos cuando no le interesa - como hace con el Reference sobre la secesión de Quebec, del TS canadiense- y seleccionando, igualmente a conveniencia, los Informes de organizaciones internacionales que le convienen, ignorando la existencia de los que no le convienen, aunque sean más directamente aplicables al caso de que trata, como hace con los Informes de la Comisión de Venecia, ignorando el crucial Informe sobre la secesión de Montenegro.

\section{C) Sobre el carácter consultivo del referéndum y sus efectos}

El planteamiento por parte de las fuerzas nacionalistas en relación con los efectos de un referéndum semejante es, cuando menos, resbaladizo. Aunque se acepta que el referéndum, jurídicamente, tiene carácter consultivo en todos los sistemas que lo aceptan, se afirma, sin embargo, que los efectos de una manifestación de la voluntad del electorado en un referéndum de este tipo, en el sentido de respaldar un estatus político diferente para la comunidad - incluida la independencia—, no pueden ser eludidos. Esta exigencia sería, a juicio del Consell Assessor per a la Transició Nacional, una consecuencia del principio democrático, entendido, ciertamente, en el mismo sentido expresado por $C i U$ en el programa electoral de 2012, como un «valor absoluto», frente al que cedería, necesariamente, cualquier otro principio político o legal (constitucional).

Las consecuencias prácticas de ese «valor absoluto» son muy significativas. El Consell lo expresa con claridad en su primer Informe. En primer lugar, a su juicio, aunque el referéndum tenga, jurídicamente, un carácter consultivo el principio democrático impondría la obligación de las instituciones representati- 
vas de actuar de acuerdo a la voluntad expresada por el electorado ${ }^{41}$. La voluntad popular así expresada impondría la reforma de la Constitución, para lo cual no necesariamente tendría que seguirse el procedimiento de reforma establecido en ella sino, alternativamente, a través de procedimientos no previstos en ella ${ }^{42}$. El principio de la eficacia al margen del Derecho, si es necesario. Una voluntad popular que, de acuerdo a lo sostenido por el Consell, no dejaría otra posibilidad que el reconocimiento de la secesión por parte del Estado, en la medida en que se considera que las instituciones de la comunidad que ha expresado esa voluntad estarían legitimadas para realizar una declaración unilateral de independencia (DUI), en caso de que no fuese asín ${ }^{43}$.

Sorprende esta construcción del Consell sobre las consecuencias del principio democrático en relación con los efectos de un referéndum consultivo. La Comisión de Venecia, en su Informe sobre buenas prácticas en materia de referéndum, diferencia claramente los efectos que hay que atribuir a los referéndums vinculantes y a los consultivos, afirmando que estos últimos no son vinculantes para las autoridades, a las que no obligan, pudiendo la institución representativa a la que le esté atribuida la competencia para adoptar la decisión actuar como considere conveniente. Es una cuestión d legalidad. Cuestión distinta, como señala la Comisión de Venencia, es el acierto político del Parlamento si opta por tomar una decisión en contra de la voluntad manifestada por el electorado ${ }^{44}$.

El Consell se refiere expresamente al Dictamen del TS de Canadá sobre la secesión de Quebec como fundamento de su posición, respecto al supuesto en que la voluntad popular fuera mayoritariamente favorable a la independencia; tanto en lo que se refiere a las obligaciones que derivarían del referéndum, a pesar de su carácter consultivo, como en lo que se refiere a los procedimientos utilizables para hacer realidad aquella voluntad. Con ello, el Consell trata de fundamentar en aquel Dictamen una posición que difiere claramente de lo que sostiene, expresamente, el alto Tribunal canadiense. En lo que se refiere a los efectos del principio democrático en relación con un referéndum consultivo sobre la secesión, el TS de Canadá, precisamente, afronta directamente la cuestión, pues la posición expresada por el Consell coincide plenamente con la que sostenían los impulsores de la secesión de Quebec — los soberanistas-. El TS canadiense afirma, en este sentido, que el argumento de que la Constitución puede ser legítimamente eludida

41 Cfr. Consell Assessor per a la Transició Nacional, cit., p. 126.

42 Cfr. Consell Assessor per a la Transició Nacional, cit., p. 128. Aunque el Consell reconoce que esta opción podría plantear problemas, considera que ello sería posible si se considera que se está ante un nuevo proceso constituyente, por lo que, de la misma forma que no está prevista la secesión y, sin embargo, habría que afrontarla, también podría afrontarse su reconocimiento al margen de la regulación constitucional vigente.

43 Cfr. Consell Assessor per a la Transició Nacional, cit., pp. 128-129.

44 cfr. European Commission for Democracy Through the Law (Venice Commission), cit., par. 43: «The foregoing does not apply to consultative referendums, which are not legally binding on the authorities. The political wisdom of Parliament going against the wishes of (the majority of) the people is clearly another matter». 
mediante el recurso a un voto mayoritario en un referéndum de ámbito territorial parece «superficialmente convincente, en gran medida porque parece invocar algunos de los mismos principios sobre los que se asienta la legitimidad de la Constitución, como son la democracia y el autogobierno». Sin embargo, continua el TS, «un análisis más detenido revela que ese argumento es erróneo, porque malinterpreta el significado de la soberanía popular y la esencia de la democracia constitucional» ${ }^{45}$. Porque la legitimidad democrática no puede reducirse a la «simple regla de la mayoría» — mucho menos si está limitada a un territorio concreto-, de forma que queden excluidos otros principios constitucionales; esto, concluye el alto Tribunal, sería un «grave error» ${ }^{46}$.

En segundo lugar, en relación con lo sostenido por el Consell, el TS de Canadá, ciertamente, afirma que el sistema constitucional «no puede permanecer indiferente» frente a una clara manifestación de voluntad a favor de la secesión. Considera que el referéndum, sin ninguna duda, puede ser un método democrático para constatar la actitud del electorado; pero la Constitución canadiense no prevé su uso «y los resultados de un referéndum no tienen un directo papel o efecto legal en nuestro esquema constitucional»; un referéndum «en sí mismo, sin nada más, no tiene efecto legal directo y no puede, por sí mismo, conducir a una secesión unilateral» ${ }^{47}$. Sobre la base de esa voluntad electoral, las instituciones del territorio estarían legitimadas para «iniciar el proceso de reforma constitucional que permita la secesión por medios constitucionales» ${ }^{48}$. Lo que significa que debe afrontarse una reforma de la Constitución, que debe ser expresa y de acuerdo al procedimiento constitucionalmente establecido para ello.

En tercer lugar, difícilmente puede encontrarse en el Dictamen del TS de Canadá respaldo a la postura que sostiene el Consell en relación con el fin ineludible de la negociación sobre la reforma constitucional. El alto Tribunal canadiense señala que está radicalmente alejado de la realidad pensar que la negociación sobre la independencia tenga un final predeterminado; en concreto, nada garantiza que termine, necesariamente, en la aprobación de esa reforma constitucional que asume la secesión del territorio. Ninguna negociación será efectiva, afirma el Tribunal, si su resultado último - la secesión - se establece como «un derecho legal absoluto» a materializarlo en la Constitución; una conclusión ineludible de ese tipo «destruiría realmente la obligación de negociar, vaciándola de contenido» (par. 91). Este rechazo a la ineludible conclusión de la negociación con la aceptación de la secesión lo asienta el TS, en primer lugar, en una razón teórica: un territorio no puede pretender invocar un derecho de autodeterminación que le permita dictar a las demás partes los términos de la secesión pretendida porque ello no sería en absoluto una negociación. En segundo lugar, en una razón prác-

45 Cfr. Reference re Secession of Quebec, cit., par. 75.

46 Cfr. Reference re Secession of Quebec, cit., par. 67.

47 Cfr. Reference re Secession of Quebec, cit., par. 87.

48 Cfr. Reference re Secession of Quebec, cit., par. 87.

UNED. Teoría y Realidad Constitucional, núm. 37, 2016, pp. 163-185 
tica: la negociación sobre la eventual reforma constitucional es una negociación muy compleja, que no se limita —como, también allí, entendían los impulsores de la secesión-, simplemente, a «los detalles logísticos de la secesión» (par. 90). Considera el tribunal que sería infantil suponer que el objetivo sustancial de la secesión pueda ser fácilmente diferenciable de los detalles prácticos de la secesión; $y$, como dice el tribunal, recordando el conocido proverbio anglosajón, «the devil would be in the details» ${ }^{49}$.

Es muy significativo que esta visión tan cautelosa sobre el desarrollo de las negociaciones sobre la reforma constitucional para tratar de afrontar la demanda de secesión la exprese el TS canadiense tras una frustrante experiencia de fallidos intentos de reforma constitucional para, precisamente, tratar de acomodar constitucionalmente las demandas políticas de la mayoría de la sociedad de Quebec. Porque las palabras del TS adquieren un significado especialmente profundo tras el fracaso de los procesos que trataron de reformar la Constitución, con los Acuerdos del Lago Meech (1987) y de Charlottetown (1992) dirigidos, precisamente, aunque de forma no coincidente, a incorporar a la Constitución canadiense la cláusula de reconocimiento de Quebec como «sociedad distinta» y sus consecuencias, en cuyo fracaso está el origen del referéndum soberanista de 1995 en Quebec y, consiguientemente, la consulta al TS y su Reference sobre la secesión de Quebec ${ }^{50}$.

Como puede comprobarse, la pretensión del Consell de justificar su posición en el Dictamen del TS de Canadá es insostenible, pues no solo simplifica hasta la caricatura la complejidad de la argumentación elaborada por aquel tribunal, sino que toma como indiscutibles interpretaciones que contradicen expresas afirmaciones contenidas en la construcción doctrinal de aquel.

Se viene diciendo que, por el contrario, el referéndum sobre la independencia de Escocia, de 2014, sí se ha planteado como un referéndum de autodeterminación ${ }^{51}$. En mi opinión, solo aparentemente. Como ya se ha dicho, el referéndum es una institución extraña a la tradición constitucional británica que, sin embargo, se ha ido abriendo camino en los últimos tiempos. Su incorporación se inserta, sin embargo, en un sistema constitucional del todo singular. Especialmente, interesa subrayar la primacía de una concepción política de la Constitución, frente a una concepción jurídica de la misma. En este contexto, el referéndum es una manifestación de la soberanía popular, que choca con el principio de soberanía parlamentaria. Así, el referéndum no se considera jurídicamente vinculante, pero sí políticamente vinculante. Lo que quiere decir que difícilmente una institución política actuará en sentido distinto al manifestado por el pueblo a través del referéndum. Así se planteó, formalmente, el referéndum sobre la independencia de

49 Cfr. Reference re Secession of Quebec, cit., par. 91.

50 Vid. A. López Basaguren: «Los referendos de secesión de Quebec...», cit.

51 Sobre el proceso del referéndum, vid. A. López Basaguren: «Escocia: referéndum sobre la independencia y propuesta de reforma de la Devolution», en Revista Española de Derecho Constitucional, n. ${ }^{\circ} 104,2015$, pp. 135-167; J. Tornos Mas: De Escocia a Cataluña. Referéndum y reforma constitucional, Madrid: Iustel 2015.

UNED. Teoría y Realidad Constitucional, núm. 37, 2016, pp. 163-185 
Escocia. Durante el debate político sobre el referéndum, que duró casi dos años, y sobre los efectos de un hipotético resultado favorable a la independencia, todas las partes manifestaron que ello supondría abrir la puerta a la independencia de Escocia. Especialmente, los partidos opuestos a la independencia insistían en que un voto mayoritario favorable a la independencia la haría ineludiblemente realidad; y los impulsores de la independencia lo corroboraban. Sin embargo, durante todo ese tiempo el debate estuvo centrado en las condiciones del acceso de Escocia a la independencia y sobre las negociaciones necesarias para hacerla posible.

En efecto, el Gobierno británico, a través de los informes de la serie Scotland Analysis, establecía unas condiciones de la hipotética Escocia independiente que eran negadas por el Gobierno escocés en su libro blanco sobre la independencia - Scotland's Future. Your Guide to an Independent Scotland - , que hacían muy difícil la viabilidad, cuando menos a corto plazo, no solo de su nacimiento como Estado independiente sino, lo más importante, el mantenimiento popular del apoyo a la independencia a pesar de que se diese un primer apoyo mayoritario a la misma. Ello era así porque el Gobierno escocés planteaba una Escocia independiente manteniendo unos especiales vínculos con el RU, considerados beneficiosos o convenientes, como el mantenimiento de la unión monetaria y otros, que, sin embargo, eran rechazados por los partidos que se oponían a la independencia. Una pretensión muy similar a la que pretendía el Gobierno de Quebec con la propuesta de partenariat con Canadá. El apoyo a la independencia podía tener posibilidades de llegar a obtener un respaldo mayoritario si se daban las condiciones por las que abogaban sus impulsores, pero que eran rechazadas por la otra parte, indispensable para que pudieran ser realidad. Lo que significa que, tras una hipotética victoria de la posición favorable a la independencia en el referéndum escocés la negociación entre las partes sobre las condiciones del acceso a la independencia eran ineludibles. Una negociación que, como señaló el TS canadiense, iba a ser extraordinariamente difícil; no, simplemente, sobre los «detalles logísticos» de la independencia sino sobre las condiciones de la misma y, por tanto, sobre su misma viabilidad. Por eso, en el RU se defendió que sería necesario un segundo referéndum sobre la independencia, una vez concluidas las negociaciones entre los representantes de Escocia y del RU sobre la independencia; es decir, una vez que el electorado escocés tuviese claras cuales eran las condiciones de acceso a la independencia. Lo que quiere decir que el referéndum escocés no era un referéndum de autodeterminación, del que se derivase automáticamente la independencia, en caso de apoyo mayoritario a esa opción.

\section{DEMANDA SECESIONISTA, PRINCIPIO DEMOCRÁTICO Y PRINCIPIO DE LEGALIDAD: ¿TIENE SALIDA EL LABERINTO?}

Las demandas secesionistas tienen un camino muy difícil en el seno de sociedades desarrolladas configuradas como sistemas democráticos. Por una parte, tie- 
nen muy difícil lograr un respaldo mayoritario que sea suficiente para pretender concluir con éxito esa trayectoria. Singularmente, por la especial dificultad de lograr el apoyo cualitativamente mayoritario de los electores que, en el contexto democrático internacional, parece legítimo exigir. Los casos de Quebec y Escocia, entre otros ${ }^{52}$, muestran esa dificultad extraordinaria en sociedades como las indicadas, incluso en condiciones de gran movilización política a favor de la independencia. En esos dos casos, a pesar de la extraordinaria movilización, el apoyo a la independencia no logró superar la mayoría simple de los votos emitidos en el referéndum. Imaginarse la hipótesis de mayorías cualificadas parece difícilmente alcanzable. Sin olvidar la falta de homogeneidad en el interior del movimiento a favor de la independencia, que puede provocar importantes problemas de gestión de esa mayoría, incluso en caso de que se produjera ${ }^{53}$.

Por otra parte, en el contexto democrático, solo parecen tener opciones de prosperar aquellos procesos a favor de la secesión que se desarrollan dentro de la legalidad y, consiguientemente, de forma negociada con el Estado del que pretenden desgajarse. Se trata de un proceso de extraordinarias dificultades. En primer lugar, porque los Estados democráticos, por norma, no contemplan constitucionalmente la posibilidad de la secesión de cualquiera de sus territorios. Pero, en todo caso, aún en los supuestos en que el Estado se preste a negociar la posibilidad de la secesión, afrontándola democráticamente, cuando exista una expresión clara del apoyo mayoritario a la secesión por parte de los ciudadanos del territorio, porque las condiciones en que puede un territorio acceder a la independencia son extremadamente difíciles de lograr en un mundo tan interdependiente. El caso de Europa es especialmente evidente en ese sentido, dada la - todavía hoy - inimaginable posibilidad de que prosperase un apoyo mayoritario a la independencia sin que el territorio se mantenga en el seno de la UE. Y, en último lugar, porque, aun así, una secesión legal solo es posible logrando el acuerdo de las instituciones del Estado del que pretende desgajarse, a los efectos de la reforma constitucional (formal o no) que, de acuerdo al estándar canadiense parece necesaria.

52 Se suele tratar muy poco el caso de Puerto Rico, territorio en el que el 6 de noviembre de 2012 se celebró un referéndum sobre su estatus político, en el que participó el 77’7\% del censo electoral. El referéndum consistía en dos preguntas. En la primera se preguntaba si se estaba de acuerdo con el mantenimiento del statu quo, es decir, que Puerto Rico siguiese siendo un Estado Libre Asociado a los Estados Unidos; votó «No» el $54 \%$ de los votantes y «Sí» el $46 \%$. En la segunda pregunta se planteaban tres alternativas posibles de cambio. La opción a favor de que Puerto Rico se convierta en un Estado integrado en los Estados Unidos (la conocida como opción por la «estatalidad») obtuvo el 61'13\% de los votos válidos (el 44’61\% del total de votos); la opción a favor de un Estado Libre Asociado soberano obtuvo el 33’32\% de los votos válidos (24’32\% del total de votos); y, la opción por que Puerto Rico sea un Estado independiente obtuvo el 5’54\% (un 4’04\% del total de votos).

53 En este sentido, es extraordinariamente ilustrativo el panorama sobre lo que se hubiera avecinado en el supuesto de que, como parecía muy probable, el sí hubiera vencido en el referéndum de 1995 en Quebec expuesto en Ch. HÉBERT —J. LAPIERRE- (2014): The morning after. The 1995 Quebec referendum and the day that almost was, Alfred A. Knopf Canada. 
Obviamente, todos estos problemas se diluyen en el supuesto de que el sentimiento favorable a la secesión sea importante en el país del que se pretende independizar el territorio. Es el caso excepcional de la división de la antigua Checoslovaquia, en la que la clase política checa vio la oportunidad de desprenderse - o librarse- de Eslovaquia, a la que muchos, en la actual República checa consideraban una rémora. Pero parece que está llamada a ser una excepción. La otra excepción, como hemos visto, es el caso de Kosovo; es decir, los supuestos de remedial secession. Pero ya hemos visto que no solo el camino a recorrer es terrible, sino que, incluso en esas circunstancias, su éxito no es sencillo.

Planteadas las cosas así, los impulsores de los procesos secesionistas claman por la insostenibilidad de un sistema semejante. Efectivamente, los procesos secesionistas como los que se están tratando en estas páginas están llamados a acabar, casi inevitablemente, en un callejón sin salida. Cuando menos, transitoriamente. A mi juicio, eso es lo que, en primer lugar, expresa la construcción doctrinal del TS canadiense en el Dictamen sobre la secesión de Quebec y lo que muestra el desarrollo de los procesos tanto en Quebec como en Escocia ${ }^{54}$.

Se trata de un callejón sin salida, a corto plazo, que solo tiene ante sí dos opciones, siempre que se mantenga dentro de los parámetros indicados. Si se pretenden romper, en uno u otro sentido, la consecuencia será la pérdida de la legitimidad en la defensa de la pretensión secesionista o en la de evitar la secesión. Es decir, si quienes pretenden la secesión consideran que ese callejón sin salida debe superarse a través de una DUI el riesgo de perder legitimidad, tanto entre parte de quienes la han respaldado — debilitando su apoyo interno- como en el ámbito exterior, principalmente internacional — debilitando extraordinariamente el respaldo que hubiera podido tener - abocarían mucho más fácilmente al fracaso su pretensión. Unas posibilidades de fracaso que serían proporcionales a la intensidad de la actitud dialogante y democrática con que se hubiera enfrentado a ellas el Estado del que pretende desmembrarse.

Creo que en la argumentación del TS de Canadá se encuentra la única opción viable que tienen a su disposición quienes promueven la secesión frente a ese callejón sin salida: cargarse crecientemente de legitimidad, sin romper las exigencias del principio de legalidad. En la argumentación del TS canadiense creo que está la convicción de que un sistema democrático no puede sostener indefinidamente una reclamación secesionista que se mantiene en el tiempo con un respaldo mayoritario constante de la población del territorio. El TS considera que la legitimidad del sistema político en ese territorio se vería seriamente afectada, lo

54 Existe un consenso muy amplio en el sentido de que el statu quo podría cambiar radicalmente en Escocia si en el referéndum que va a celebrarse en junio en el RU sobre la permanencia o la salida de la UE se produjera una victoria de los favorables al Brexit si, al mismo tiempo, como parece probable, una mayoría del electorado escocés votase a favor de la permanencia. Esa circunstancia abriría la puerta a un nuevo referéndum sobre la independencia en Escocia que, muy probablemente, cambiaría las condiciones cualitativas que rodean esta cuestión. 
que acabaría siendo democráticamente insostenible y obligaría a actuar a las instituciones del resto del país. La relación que establece entre legalidad y legitimidad obliga, ciertamente, al respeto de la legalidad por parte de quienes pretenden la secesión; pero obliga a quienes pretenden garantizar que la legalidad no sea conculcada a garantizar que esa legalidad esté permanentemente revitalizada en su legitimidad.

El sistema democrático corre serios riesgos, que trascienden la cuestión de la secesión, si opta por una actitud de pasivo atrincheramiento en la legalidad, tal y como se ha señalado. Si quienes propugnan la secesión no tienen otra opción que la de tratar de mantener o incrementar el apoyo a la misma por la población del territorio y cargarse crecientemente de legitimidad, hasta hacer insostenible en un sistema que quiera seguir siendo saludablemente democrático el rechazo a la reforma que permitiera la secesión legalmente, el Estado debe optar por afrontar los problemas que están en la base de la fortaleza del apoyo social a la reclamación secesionista ${ }^{55}$.

Los sistemas que no han sido capaces de adentrase por la vía de una reforma del sistema de autonomía territorial, de forma que se trate de dar una respuesta suficientemente satisfactoria para una parte importante de quienes, en un momento determinado, pero no en cualquier circunstancia, están dispuestos a respaldar la reclamación secesionista, son sistemas que fracasan. Es el caso del Reino Unido y la independencia de Irlanda. Solo han sido capaces de gestionar adecuadamente las demandas de secesión aquellos países que encaran con acierto esa reforma, logrando una satisfacción suficiente en la integración en el sistema por una parte cualitativamente importante de quienes en un momento de crisis política han apoyado las demandas de secesión. Es el caso, hoy por hoy, de Canadá respecto a Quebec ${ }^{56}$.

En España nos encontramos con el entrecruzamiento de dos errores. El error de los secesionistas, que creen que el principio democrático les otorga un aval suficiente para romper la legalidad si el Estado no accede a sus pretensiones y el error del sistema político, del Estado, que cree que la legalidad es una trinchera infranqueable, por lo que no hay necesidad de afrontar la reforma del sistema autonómico para tratar de dar solución a los problemas que han permitido a los impulsores de la demanda secesionista lograr un apoyo cualitativamente muy importante de la sociedad catalana.

55 En esta dirección, son muy interesantes las reflexiones contenidas en el último capítulo del trabajo de J. Tudela Aranda: El fracasado éxito del Estado autonómico. Una historia española, Madrid: Marcial Pons, 2016, pp. 285 y sigs.

56 Vid., más en detalle, sobre la incapacidad del RU para afrontar la reforma del sistema en torno a las demandas de Home Rule para Irlanda entre finales del siglo XIX y principios del siglo xx, por una parte, y la forma en que el sistema federal canadiense ha afrontado el acomodamiento de Quebec, A. López BASAGUREN (2015): «Sobre el desarrollo federal del sistema autonómico», en García Herrera, M.A.; Asensi SABATER, J.; Balaguer Callejón, F. (coords.): Constitucionalismo crítico. Liber Amicorum Carlos de Cabo Martín, Valencia: Tirant lo Blanch, 2015. 
En estas condiciones, los secesionistas seguirán teniendo muy difícil lograr sus pretensiones. Pero el riesgo de profundo deterioro del sistema democrático es extraordinario.

TitLe: The Secession Claim in Catalonia and the Democratic System. The «Procés» from a Comparative point of view

ABSTRACT: This paper analyzes the political process of the independence demand in Catalonia and, specifically, the argument of the democratic principle as constitutional basis of the right to decide the future of Catalonia. This paper also analyzes the comparative experience of other countries, particularly Canada, where there have been similar demands and with the same argument.

RESUMEN: Este trabajo analiza el proceso político en torno a la demanda de secesión que se viene desarrollando en Cataluña y, en concreto, el argumento del principio democrático en el que los demandantes de la autodeterminación se apoyan para fundamentar su derecho a decidir su futuro como comunidad política. Este análisis se realiza a la luz de la experiencia de otros países, especialmente de Canadá, en los que se han dado demandas similares y con ese mismo argumento.

KEY WORDS: Catalonia, secession claim, right to decide, democratic principle, comparative law.

Palabras Clave: Cataluña, demanda de secesión, derecho a decidir, principio democrático, derecho comparado.

FECHA DE RECEPCIÓN: 11.12.2015 FECHA DE ACEPTACIÓN: 15.01.2016 